\title{
Cities as biological computers
}

Claudia Pasquero and Marco Poletto

In this article, we propose a conceptual model and a computational design method to articulate the world's Urbansphere, suggesting new terms for its sustainable coevolution with the Biosphere. The proposed model responds to principles of biological self-organisation, and operates by embedding a numerical/computational engine, a living Physarum polycephalum grown on a spatial/morphogenetic substratum and a Satellite-driven informational territory.

The article envisions a future when manmade infrastructures and non-human biological systems will constitute parts of a single biotechnological whole. In this respect the article can be read as a manifesto for the extension of biotechnology to the scale of the Biosphere (through geoengineering) by expanding the scope and material articulation of global informational and energetic infrastructures (the internet of things and the internet of energy).

\section{Urbansphere versus Biosphere}

Accordingly to the Global Footprint Network, on the 13 August 2015 Earth had hit what is known as the Earth Overshoot Day. This means that Earth had exhausted its budget of resources for the year within eight months. For the remaining days the ecological deficit is maintained by drawing down local resource stocks and accumulating carbon dioxide in the atmosphere, in other words we will be operating in overshoot.(1)

This operational deficit is made possible by the existence of what the authors call the Urbansphere, the global apparatus of contemporary urbanity, a dense network of informational, material, and energetic infrastructures that sustain our increasingly demanding urban metabolisms while providing a buffer with the fluctuations and deficiencies of the natural Biosphere.

There is, we will suggest, a latent potential to reframe sustainability as an urban design challenge, ultimately envisioning a future where manmade infrastructures and non-human biological systems will constitute part of a single biotechnological whole.

The article will also suggest an application of the model to a specific case study demonstrating its efficacy in the reconceptualisation of the postindustrial and ecologically depleted landscapes of eastern Arizona.

\section{The Physarium machine}

The biocomputational model presented in the article is embodied in the Physarum Machine, a biodigital apparatus conceived by the authors and further developed within the Urban Morphogenesis Lab at the UCL in London.

The use of specifically designed apparatus of material computation to demonstrate and solve problems of urban morphogenesis is not new and the authors have taken great inspiration for the conception of the Physarum machine in the work of German Architect Frei Otto. 
Otto, famously, studied processes of form generation and diagrams of occupation and connection for large territories by means of the computational power of soap bubbles, sand, ink droplets, and other materials.

Material computation operates morphologically and in relationship to its specific substratum or medium. Otto was able to compute in a completely analogical way the emergence of path systems and, in particular, to define a special category of path systems, the so-called minimising detour networks (2).

Minimising detour networks are special among others as they produce connections among points that are optimal in terms of the energy expenditure required to connect them. This notion of energy implies the existence of a substratum and that of an 'organism' that moves over it. Otto discovered that the patterns emerging from his experiments appeared almost everywhere in non-human as well as in human systems, such as in settlements and road networks.

He famously stated:

The settlement, the human city, is natural. To recognise it is a natural science. To tend it is an art, analogous to horticulture. This cannot exist without knowledge of the plants, soil and water involved. The art of urban development requires knowledge of all living organisms in nature, of non-living nature, the present state and the possibilities of technology.(3)

Frei Otto's intuition seems more relevant today in the light of what we described before as the necessity to find models of co-evolution of our Urbansphere, evolved into an increasingly complex but also rigid and fragile network, and its dynamic substratum, the Biosphere.

Contemporary technology is offering renewed means to operate in this territory, with biotechnology allowing us not only to mimic nature but to actually hack into nature's codes and augment its functionality. Information technology in turn, is enabling the emergence of a global informational network connecting all things human and nonhuman, living and non-living (IoT).

These influences have led to the development of the Physarum machine, characterised by the crafted juxtaposition of a digital substratum of communication and a biological computer. The main driver of this purposeful hybridisation has been the attempt to explore and represent within a small-scale apparatus this notion of 'naturalness' as observed by Otto, beyond the living biological world of Nature.

If on the one side the machine inherits this approach to a form of 'natural urbanism', the other also grows out of the realisation that the living biosphere is, today, understood as the product of human technology and science, and therefore is no longer natural.

This idea was first developed by the authors when visiting two years ago (2014) the site of the greatest experiment in biospheric science of all time, Biosphere 2, near Tucson in Arizona. The site, now converted into a research centre for the University 
of Arizona, once hosted the most complex selfcontained reconstruction of the Earth's biosphere ever built. As we approached the main Biosphere 2 greenhouse, the white steel space frame structure piercing through the thick vegetation in the forest biome, clearly visible, immediately caught our attention.

The forest biome was once connected spatially with the ocean and desert biomes, reflecting the continuity within the real biosphere. These five biomes would, in fact, interact and self-organise, generating multiple transitions zones or, biotones.

According to early theories of biospheric self-organisation, allowing such a degree of freedom of differentiation would increase the resilience of the system, and possibly its ability to absorb $\mathrm{CO} 2$, produce oxygen, filter water, and grow edible food.

The original goal of the project was to create a self-contained space where people could survive in a self-sufficient manner (and indeed eight of them, the biospherians, did live for two years inside the structure). It was about investigating the possibility of recreating our biosphere inside an enclosed artificial system, a manmade apparatus.(4)

Today, thirty years later, we can think of this unique experiment as taking place, in real-time, within Biosphere 1, a term reserved for the Earth's own biosphere. Let's just consider for a moment, our globalised and interconnected world, where flows of material, energy, and information that feed our contemporary cities now span continents. They connect points across Biosphere 1 as part of a resource-driven industry that is both necessary to the survival of our urbanising society and, potentially, detrimental to the wealth and wellbeing of its inhabitants. Now, let us consider that these connections comprise a global Urbansphere thatwraps the world over and extensively exchanges with the earth's biosphere at an incredible rate. The paradox is that while this Urbansphere has increasingly become our preferred habitat, it is also causing the greatest threats to our survival.

The model presented in this article and embodied in the Physarum machine, proposes a solution to this seemingly unsolvable paradox by redefining the conceptual separation of the Urbansphere from the biosphere. The two are now so intricately connected and coevolutionary they cannot be separated. We therefore propose to remodel the Urbansphere as an augmented biosphere.

How can we conceptualise and model this Urbansphere so that it can evolve into a resilient and adaptive habitat for humanity and the other species connected to the biosphere?

\section{The SLIME mould: from biological organism to biodigital analogue}

The slime mould, or in scientific terms the Physarum Polycephalum, was chosen as computational model; the reason for this choice is in the unique nature of this bizarre creature and in the multiplicities of uses that its behaviour has inspired in scientists, programmers, and engineers alike.

The slime mould is a protist, in fact it is a single cell, just one cell, but a very peculiar one. Within this extraordinary body we can find thousands if not millions of nuclei that float in protoplasmic liquid. 
The collective is kept together by an actin membrane. In the plasmodium phase when enough nutrients are to be found the membrane stretches to take virtually any morphology. The nuclei interact with each other and the environment via chemical reactions that generate gradients of pressure that regulate the protoplasmic flows. In this way the organism can locally sense the presence and amount of nutrients in a given place and time, and react to them. This reaction is locally driven and based on multiple random interactions that is unaided by central planning, or decision-making.

The slime mould is a special kind of biological computer that has no discrete brain but is able to leave traces in the environment that constitute a form of spatial and distributed memory. This memory enables the slime mould to develop and optimise its behaviours.

Scientists experimenting with slime mould discovered that this simplest of all collective organisms can in fact perform extremely sophisticated tasks such as network optimisations, nutrient regulation, and may even anticipate events.(5) So, the mould not only represents a simple form of bottom up self-organisation via multiple interactions, but also is capable of performing multiple forms of computation and optimisation without recurring to a centralised brain. Also, these kinds of optimisations are recurrent in any collective organism and are critical to its survival. Urban systems also demonstrate optimisation behaviours; after all cities too must adjust the way they move around goods and energy, need to regulate the amounts they extract from each extraction/ production site, and are always trying to predict or anticipate daily, seasonal, or epochal fluctuations.

It is known in fact that transport engineers and computer scientists already took notice and adopted slime mould in a set of experiments developed to simulate traffic infrastructures across entire continents.(6)

We will now explore the optimisation strategies through which slime mould achieves its goals. It turns out, these are unlike any planned urban systems as they are purely the product of emergent collective behaviour and distributed spatial memory.

\section{The Physarum Machine Apparatus}

For the slime mould to offer a relevant design model of the Urbansphere, as we defined it before, it must be embedded in an augmented substratum, in direct communication with the earth biosphere.

To test this idea, the Physarum machine was designed as a new kind of apparatus to embed the slime mould onto a prototypical scenario, in this instance the copper mining corridor in Arizona, US.

The apparatus creates a hybrid, interconnected, and unstable substratum of growth for the slime mould, which enables us to feed the slime mould with data from the territory of choice and to capture and visualise the behaviour of the slime mould itself.

INPUT: we have 3 kinds of input parameters that communicate with the slime mould - substratum morphology, light fields, and food points. 


\section{Substratum morphology}

Topographic and morphologic information is translated into 3D-printed substrata, which represent morphological boundaries of matter in the real world and become the basis for the development of the slime mould's spatial memory. The material therefore needs to capture the traces left by the slime mould and store them in time. In the last version of the machine this substratum was 3D-printed in ABS plastic and coated by a thin layer of agar.

\section{Light fields}

Gradients of light are produced by an LED matrix that the slime mould seeks to avoid. These represent areas of friction or obstacles in the real world and can change over time as a manifestation of live data streams.

\section{Food points}

Nutrient particles that drive the slime mould's metabolism are provided as it tries to reach and exploit them. At any given moment the mould adjusts its morphology and develops its distributed intelligence, while minimising the expenditure of energy to complete the task. These events represent opportunities and resources or sources of energy in the real world. In the last version of the Physarum

Machine, these are delivered by means of a customised 3D printer, which is capable of delivering in the exact location of study a minute quantity of nutrients.

In the experiment described in this article the slime mould is kept wet and food is dropped in the locations of present and future mining. Such locations have been retrieved from a satellite survey where crosses mark the points of current drilling for copper as well as a distribution of testing boreholes that resulted in sufficient amounts of copper ore to justify future drilling. It must be noted that the peculiar nature of the copper corridor means copper ore is diffused in the territory at very low density. This makes open pit mining obligatory and also forces a sprawling model of extraction that leaves large areas of landscape scarred and generates a large amount of detritus that is unusable for the mining industry. Therefore, it becomes sediment in large tailing ponds that further modify the original morphology of the territory.

In other words, small quantities of valuable resources are scattered on a large terrain, which provided the perfect testing ground for the sensitive slime mould to compute.

In our experiment the source of nutrient for the slime mould was also colour coded with ink according to the specific mineral content and status of the mining location. As the mould expands to reach out for food it forms a network and begins dissolving and distributing the nutrients along it. Since its walls are transparent, the colour in the nutrients affects the colour of the slime mould and allows us to directly observe the computational processes taking place within the slime mould's body. While some scientists are suggesting this approach could be one of the main mechanisms for the development of future biological computers, the technique is also a way of demonstrating how networks actually transport matter. 
A high-resolution webcam located on top of the machine captures the mould's behaviour, morphology, and the colour nuances at any moment.

At time zero the slime mould is introduced in the machine at three specific points; from these locations it starts spreading in search of food. At this stage we record slime mould behaviours as droplets of nutrients are introduced in mining locations while the LED lighting pattern reproducing site obstacles is activated.

The slime mould's behaviour is based on an internal algorithm that optimises the expenditure of energy to reach out for nutrients. It biologically computes this task in relationship to the amount of nutrients available at each moment in time and their necessity to be distributed to reach all the nuclei in the cell. As the mould begins exploiting the nutrients it visibly modifies the landscape producing a network of traces that improve its ability to compute the challenge. In other words, it becomes successful in minimising the overall length, or volume of its networked body to connect effectively the most profitable sources of nutrients. However, this solution is reached only after a long scanning sequence where the mould tests multiple solutions or paths to connect the desired location and evaluate their relevance against to the overall metabolic balance. Only after much searching the emergent process is able to select the best paths and drop the others. This may initially seem a terribly wasteful process but its potential becomes clear as the experiment progresses.

The paths that are discarded in fact remain in the landscape as traces. These accumulate and constitute a form of distribute spatial memory. As resources are consumed the mould adjusts its morphology to rebalance its metabolism. This adjustment is made with further iterations of its search and select mechanism. However, these iterations become more and more effective as the amount of traces increases. A point is reached where the distributed memory allows the mould to 'take real-time decisions' about how to optimise its morphology and rebalance resources. It may also even anticipate their scarcity, or frequency of renewal.

This form of embedded memory transforms and incorporates the substratum into the slime mould computational body. The final result is captured in the distribution of colours. This form of abstract biodigital painting encodes the morphogenesis of an emergent landscape, transformed in time by the interaction with the living 'infrastructure' of the slime mould. This transformation of matter depicts the encoding of a distributed memory (information) for a bottom-up algorithm to continuously optimise the delivery and usage of available resources (energy).

\section{Synthesis}

This has a critical significance in opening up a new vision for the Urbanasphere, or future of our cities. The Physarum machine provides an operational model for recasting the relationship between urban infrastructure and landscape. The necessity for such reconsideration is evident in our resource-driven contemporary urbanisation and can be exemplified by case studies such as the manufactured landscapes of Arizona, as well as many resource-driven boomtowns around the globe. 
In the experiment we also looked at ways of translating this biological computational process into drawings, and forms of semi-autonomous collective machines. We have captured high resolution images of the experimental terrain. An algorithm that can recognise the morphology of the slime mould in time and simulate the swarm of nuclei as they move along its branches has also been developed.

Our virtual agents that have been scripted in Processing on the basis of a modified swarm intelligence model with the ability to read and write a background map, also leave a trace of their movement. Traces accumulate as the experiment goes on and lines of movement become more stable.

In these emergent drawings a beautiful kind of fuzziness is produced - their edges being in continuous fluctuation.

The code translated this logic to a swarm of excavating machines, thus redescribing the mining landscape of Arizona into an evolving, self-aware territory of excavation and reshaping. Zooming from territorial to human scale reveals more detail but never a fixed, neat boundary.

Feeding back these findings into the initial analyses of the territory of the copper corridor in Arizona and its past, present and future developments, we can transform its fundamental relationship to the resources it has been set up to extract and the scarred landscape it typically leaves behind.

\section{Conclusion}

This article proposes how biological computing aided by design intuition could have a radical effect on the way we conceive cities. In doing so, it redescribes an ongoing relationship between the Urbansphere and Biosphere.

From a methodological point of view biosciences are used to investigate how we can go beyond digital as well as descriptive simulations and how we could start working with material to define new methods of computing.

We believe that there is an interest in augmenting material through sensors and microprocessors so that we could start harvesting the computational power present in micro-organisms like the slime mould. We dream of an era where descriptive computation will be overcome and computer will become superseded by our capability to simulate and compute through the world that surrounds us.

From a social and cognitive point of view we look at pre-industrial society and the relationship they have established with natural systems, their ability to communicate with the world surrounding them - and how we could recuperate this with a different consciousness.

Prehistoric communities that interrogated nature before extracting resources from a site may have used a similar set of procedures that may have been thought of as 'magical rituals'. Today, we might define similar processes as 'pattern recognition' or self-organising experiments. 
In a similar manner in pre-industrial community in Europe the population was able to predict events such as weather, insect arrivals, etc. by reading signs in the environment surrounding them. When we lost this capability, we became consumers.

By applying such novel computational systems, the capability of the single individual in our city to read matter and processes around us may change completely from consumer to creator. This could trigger self-organising processes that would enable cities to grow as a result of interaction rather than as a result of planning and engineering. Potentially, this could lead to a complete redescription of the relationship between Urbansphere and Biosphere.(7)

Notes

1. Published by the Global footprint network on the following webpage: <http://www.footprintnetwork.org/en/index.php/GFN/page/earth_overshoot_day> (accessed 1 January 2016).

2. Marek Kolodziejczyk, Wood-Thread Model to Compute Optimised Detour Path Network, Institute for Lightweight Structures (Stuttgart: ILEK 1991).

3. Otto Frei, Occupying and Connecting (Stuttgart: Axel Menges 2009), p. 111.

4. John Allen, Me and the Biospheres: A Memoir by the Inventor of Biosphere 2 (Santa Fe: Synergetic Press, 2009).

5. Chris R. Reid, Madeleine Beekman, Tanya Latty, Audrey Dussutour, 'Amoeboid Organism Uses Extracellular Secretions to Make Smart Foraging Decisions', in The Official Journal of the ISBE International Society for Behavioural Ecology, Advance Access Publication (18 April 2013), DOI:10.1093/beheco/ art032. [accessed 16. 02.16]

6. See also: A. Tero, S. Takagi, T. Saigusa, K. Ito, D. P. Bebber, M. D. Fricker, K. Yumiki, R. Kobayashi, T. Nakagaki, 'Rules for Biologically Inspired Adaptive Network Design', in Science, 327:5964 (2010), p. 439.

7. For more on interfacing Slime mould with autonomous robots see: Soichiro Tsudaa, Klaus-Peter Zaunerb, Yukio-Pegio Gunjia, 'Robot Control with Biological Cells, 15 July 2006', in BioSystems, 87 (2007), pp. 215-23. 